\title{
Entire solutions for a class of variational problems involving the biharmonic operator and Rellich potentials
}

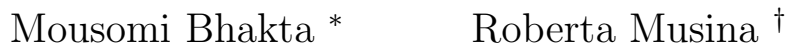

\begin{abstract}
We study existence, multiplicity and qualitative properties of entire solutions for a noncompact problem related to second-order interpolation inequalities with weights. More precisely, we deal with the following family of equations

$$
\Delta^{2} u=\lambda|x|^{-4} u+|x|^{-\beta}|u|^{q-2} u \quad \text { in } \mathbb{R}^{N},
$$

where $N \geq 5, q>2, \beta=N-q(N-4) / 2$ and $\lambda \in \mathbb{R}$ is smaller than the Rellich constant.

Keywords: Caffarelli-Kohn-Nirenberg type inequalities, weighted biharmonic operator, dilation invariance, breaking positivity, breaking symmetry.

2010 Mathematics Subject Classification: 26D10, 47F05.
\end{abstract}

\section{Introduction}

In this paper we study weak solutions to problem

$$
\left\{\begin{array}{l}
\Delta^{2} u=\lambda|x|^{-4} u+|x|^{-\beta}|u|^{q-2} u \quad \text { in } \mathbb{R}^{N} \\
u \in \mathcal{D}^{2,2}\left(\mathbb{R}^{N}\right), \quad u \neq 0
\end{array}\right.
$$

Our main assumptions are the following: $N \geq 5$ and

$$
q>2, \quad \lambda<\gamma_{N}^{2}, \quad \beta=N-q \frac{N-4}{2}
$$

where

$$
\gamma_{N}:=\frac{N(N-4)}{4} .
$$

${ }^{*}$ TIFR Centre for Applicable Mathematics, Post Bag No. 6503 Sharadanagar, Chikkabommasandra, Bangalore 560065. Email: mousomi@math.tifrbng.res.in

${ }^{\dagger}$ Dipartimento di Matematica ed Informatica, Università di Udine, via delle Scienze, 206 - 33100 Udine, Italy. Email: musina@uniud.it. Partially supported by Miur-PRIN 2009WRJ3W7-001 Fenomeni di concentrazione e problemi di analisi geometrica 
It is well known that $\gamma_{N}^{2}$ is the best constant in the Rellich inequality

$$
\int_{\mathbb{R}^{N}}|\Delta u|^{2} d x \geq \gamma_{N}^{2} \int_{\mathbb{R}^{N}}|x|^{-4}|u|^{2} d x \quad \text { for any } u \in \mathcal{D}^{2,2}\left(\mathbb{R}^{N}\right)
$$

see [12, [13].

Some existence and multiplicity results for (1.1) in case $\lambda=0$ can be found in [4]. If $q=2^{* *}$, where

$$
2^{* *}:=\frac{2 N}{N-4}
$$

is the critical Sobolev exponent, then (1.1) becomes

$$
\left\{\begin{array}{l}
\Delta^{2} u=\lambda|x|^{-4} u+|u|^{2^{* *}-2} u \quad \text { in } \mathbb{R}^{N} \\
u \in \mathcal{D}^{2,2}\left(\mathbb{R}^{N}\right), \quad u \neq 0 .
\end{array}\right.
$$

In particular, if in addition $\lambda=0$ then ground state solutions to (1.5) are extremals for the Sobolev constant $S^{* *}$ relative to the embedding $\mathcal{D}^{2,2}\left(\mathbb{R}^{N}\right) \hookrightarrow L^{2^{* *}}\left(\mathbb{R}^{N}\right)$, see for instance [14]. The second-order version of (1.5), namely,

$$
\left\{\begin{array}{l}
-\Delta u=\lambda|x|^{-2} u+|u|^{2^{*}-2} u \quad \text { in } \mathbb{R}^{N} \\
u \in \mathcal{D}^{1,2}\left(\mathbb{R}^{N}\right), \quad u \neq 0
\end{array}\right.
$$

was studied by Terracini [15], under the assumption $\lambda<(N-2)^{2} / 4$, the Hardy constant. We quote also [5], [9] and references there-in for a larger class of problems related to the Caffarelli-Kohn-Nirenberg inequalities [2].

In the first part of the paper we deal with radially symmetric solutions to (1.1). By using the Rellich inequality (1.4) and the results in [4] one can check that for any $q, \lambda$ and $\beta$ satisfying (1.2), the infimum

$$
S_{q}^{\mathrm{rad}}(\lambda):=\inf _{\substack{u \in \mathcal{D}^{2,2}\left(\mathbb{R}^{N}\right) \\ u=u(|x|), u \neq 0}} \frac{\int_{\mathbb{R}^{N}}|\Delta u|^{2} d x-\lambda \int_{\mathbb{R}^{N}}|x|^{-4}|u|^{2} d x}{\left(\int_{\mathbb{R}^{N}}|x|^{-\beta}|u|^{q} d x\right)^{2 / q}}
$$

is positive. The following existence result holds.

Theorem 1.1 Let $N \geq 5, q>2, \lambda<\gamma_{N}^{2}$, and put $\beta=N-q(N-4) / 2$. Then problem (1.1) has at least one radially symmetric solution $u$ that achieves $S_{q}^{\mathrm{rad}}(\lambda)$.

Notice that problem (1.1) is invariant with respect to the weighted dilation

$$
u(x) \mapsto t^{\frac{N-4}{2}} u(t x), \quad t>0 .
$$

From now on we will identify solutions that coincide up to a weighted dilation and change of sign. This agreement is used in the next uniqueness and positivity result. 
Theorem 1.2 Let $N \geq 5, q>2$, and put $\beta=N-q(N-4) / 2$. If

$$
-(N-2)^{2} \leq \lambda<\gamma_{N}^{2}
$$

then there exists a unique radially symmetric solution $u \in \mathcal{D}^{2,2}\left(\mathbb{R}^{N}\right)$ to (1.1). Moreover, $u$ is positive on $\mathbb{R}^{N}$.

Theorems 1.1 and 1.2 will be proved in Section 3. Some preliminary results of independent interest will be proved in Section 2 .

Notice that the positivity of $u$ does not follow from its characterization as an extremal for $S_{q}^{\mathrm{rad}}(\lambda)$. Indeed, in problems involving the biharmonic operator a breaking positivity phenomenon might appear and ground state solutions might be forced to change sign, see for instance [4]. Actually one could wonder whether extremals for $S_{q}^{\mathrm{rad}}(\lambda)$ change sign if $\lambda<<0$. A similar phenomenon would be completely new with respect to (1.6) and to similar second-order problems.

In case $q \leq 2^{* *}$ one can use again variational methods to find solutions to (1.1) that are not necessarily radially symmetric. We recall that

$$
\int_{\mathbb{R}^{N}}|\Delta u|^{2} d x \geq S^{* *}\left(\int_{\mathbb{R}^{N}}|u|^{2^{* *}} d x\right)^{2 / 2^{* *}} \quad \text { for any } u \in \mathcal{D}^{2,2}\left(\mathbb{R}^{N}\right),
$$

where $S^{* *}$ is the Sobolev constant. If $q$ and $\beta$ are as in (1.2), then by interpolating (1.8) and (1.4) via Hölder inequality, one plainly gets that there exists a constant $C=C(N, q)>0$ such that

$$
\int_{\mathbb{R}^{N}}|\Delta u|^{2} d x \geq C\left(\int_{\mathbb{R}^{N}}|x|^{-\beta}|u|^{q} d x\right)^{2 / q} \quad \text { for any } u \in \mathcal{D}^{2,2}\left(\mathbb{R}^{N}\right) .
$$

Notice that (1.9) is the second-order version of the celebrated Caffarelli-Kohn-Nirenberg inequalities [2]; we quote also [4] for a large class of dilation-invariant inequalities on cones. It is clear that the infimum

$$
S_{q}(\lambda):=\inf _{\substack{u \in \mathcal{D}^{2,2}\left(\mathbb{R}^{N}\right) \\ u \neq 0}} \frac{\int_{\mathbb{R}^{N}}|\Delta u|^{2} d x-\lambda \int_{\mathbb{R}^{N}}|x|^{-4}|u|^{2} d x}{\left(\int_{\mathbb{R}^{N}}|x|^{-\beta}|u|^{q} d x\right)^{2 / q}}
$$

is positive, provided that $\lambda<\gamma_{N}^{2}$. In addition, extremals for $S_{q}(\lambda)$ give rise to solutions to (1.1). In Section 4 we prove the next existence result.

Theorem 1.3 Let $N \geq 5, q \in\left(2,2^{* *}\right], \lambda<\gamma_{N}^{2}$, and put $\beta=N-q(N-4) / 2$.

(i) The infimum $S_{q}(\lambda)$ is achieved for any $q \in\left(2,2^{* *}\right)$.

(ii) The infimum $S_{2^{* *}}(\lambda)$ is achieved if and only if $\lambda \geq 0$. 
Clearly enough, it results that $S_{q}(\lambda) \leq S_{q}^{\mathrm{rad}}(\lambda)$. In Section 5 we wonder whether the solutions in Theorem 1.3 are radially symmetric or breaking symmetry occurs. First, by using rearrangement techniques we prove that $S_{q}(\lambda)=S_{q}^{\mathrm{rad}}(\lambda)$ provided that $\lambda \geq 0$. In contrast, we show that if $\lambda<<0$ then $S_{q}(\lambda)<S_{q}^{\text {rad }}(\lambda)$. Therefore, if in addition $q \in\left(2,2^{* *}\right)$ then problem (1.1) has at least two distinct solutions.

\section{Notation}

We put $\mathbb{R}_{+}=(0, \infty)$ and we denote by $B_{R}$ the open ball in $\mathbb{R}^{N}$ of radius $R>0$ centered at 0 .

Let $\omega$ be a non-negative measurable function on a domain $\Omega$ in $\mathbb{R}^{n}$. For any $p \in[2, \infty)$ the weighted Lebesgue space $L^{p}(\Omega ; \omega(x) d x)$ is the space of measurable maps $u$ in $\Omega$ with finite norm $\left(\int_{\Omega}|u|^{p} \omega(x) d x\right)^{1 / p}$. If $\omega \equiv 1$ we simply write $L^{p}(\Omega)$.

Let $N \geq 5$. The space $\mathcal{D}^{2,2}\left(\mathbb{R}^{N}\right)$ is the completion of $C_{c}^{\infty}\left(\mathbb{R}^{N}\right)$ with respect to the Hilbertian norm

$$
\|u\|^{2}=\int_{\mathbb{R}^{N}}|\Delta u|^{2} d x .
$$

Then $\mathcal{D}^{2,2}\left(\mathbb{R}^{N}\right) \hookrightarrow L^{2}\left(\mathbb{R}^{N} ;|x|^{-4} d x\right)$ by the Rellich inequality (1.4), and $\mathcal{D}^{2,2}\left(\mathbb{R}^{N}\right) \hookrightarrow L^{2^{* *}}\left(\mathbb{R}^{N}\right)$ by Sobolev embedding theorem, where $2^{* *}=2 N /(N-4)$. We denote by $S^{* *}$ the (second order) Sobolev constant, that is,

$$
S^{* *}=\inf _{\substack{u \in \mathcal{D}^{2,2} u\left(\mathbb{R}^{N}\right) \\ u \neq 0}} \frac{\int_{\mathbb{R}^{N}}|\Delta u|^{2} d x}{\left(\int_{\mathbb{R}^{N}}|u|^{2^{* *}} d x\right)^{2 / 2^{* *}} .}
$$

It is well known that $S^{* *}$ is achieved by a (unique, up to a multiplicative constant, and up to dilations and translations in $\mathbb{R}^{N}$ ) positive and radially symmetric function.

If $g \in L^{1}(\mathbb{R})$ we will often use the notation $\int g d s$ instead of $\int_{-\infty}^{\infty} g(s) d s$.

\section{Homoclinic solutions to a fourth order ODE}

To prove Theorems 1.1 and 1.2 we need some preliminary results that hold for ordinary differential equations involving differential operators of the form

$$
L w=w^{\prime \prime \prime \prime}-2 A w^{\prime \prime}+B^{2} w .
$$

We start with a lemma about a linear equation.

Lemma 2.1 Let $a \in L^{\infty}, a \geq 0$ and let $\eta \in H^{2}\left(\mathbb{R}_{+}\right)$be a solution to

$$
\left\{\begin{array}{l}
\eta^{\prime \prime \prime \prime}-2 A \eta^{\prime \prime}+B^{2} \eta=a(s) \eta \quad \text { on } \mathbb{R}_{+} \\
\eta(0)=\eta^{\prime}(0)=\eta^{\prime \prime}(0)=0 .
\end{array}\right.
$$

If $A \geq B \geq 0$ then $\eta \equiv 0$ on $\mathbb{R}_{+}$. 
Proof. If $\eta^{\prime \prime \prime}(0)=0$ then we are done, since we immediately infer $\eta \equiv 0$. We argue by contradiction. Replacing $\eta$ with $-\eta$ if needed, we can assume that $\eta^{\prime \prime \prime}(0)>0$. Since $A \geq B$, then the equation $c^{2}-2 A c+B^{2}=0$ has two real nonnegative roots $c_{+}, c_{-}$. Next we put

$$
\psi=-\eta^{\prime \prime}+c_{+} \eta
$$

Notice that in particular $\psi \in H^{1}\left(\mathbb{R}_{+}\right)$and it solves

$$
\left\{\begin{array}{l}
-\psi^{\prime \prime}+c_{-} \psi=a(s) \eta \quad \text { on } \mathbb{R}_{+} \\
\psi(0)=0 \\
\psi^{\prime}(0)<0
\end{array}\right.
$$

Since $6 \eta(s)=\eta^{\prime \prime \prime}(0) s^{3}+o\left(s^{3}\right)$ and $\psi(s)=-\eta^{\prime \prime \prime}(0) s+o(s)$ as $s \rightarrow 0^{+}$, and since $\eta^{\prime \prime \prime}(0)>0$, then $\eta>0$ and $\psi<0$ in a right neighborhood of 0 . In addition, $\eta$ is smooth and vanishes at infinity. Thus there exists $s_{\infty} \in(0, \infty]$ such that $\eta>0$ in $\left(0, s_{\infty}\right)$ and $\eta \in H_{0}^{1}\left(0, s_{\infty}\right)$. We claim that $\psi<0$ on $\left(0, s_{\infty}\right)$. If not, there exists $s_{1} \in\left(0, s_{\infty}\right)$ such that $\psi<0$ on $\left(0, s_{1}\right)$ and $\psi \in H_{0}^{1}\left(0, s_{1}\right)$. But then from (2.3) we readily get

$$
\int_{0}^{s_{1}}\left|\psi^{\prime}\right|^{2}+c_{-} \int_{0}^{s_{1}}|\psi|^{2}=\int_{0}^{s_{1}} a(s) \eta \psi \leq 0
$$

which is impossible. Finally, using $\eta \in H_{0}^{1}\left(0, s_{\infty}\right)$ as test function in (2.2) and recalling that $\eta>0$ and $\psi<0$ on $\left(0, s_{\infty}\right)$, we get

$$
\int_{0}^{s_{\infty}}\left|\eta^{\prime}\right|^{2}+c_{+} \int_{0}^{s_{\infty}}|\eta|^{2}=\int_{0}^{s_{\infty}} \eta \psi \leq 0
$$

a contradiction. The Lemma is completely proved.

Next we state the main result of this section.

Theorem 2.2 Let $q>2$ and let $A, B$ be two given constants, such that $A, B>0$.

(i) The ordinary differential equation

$$
w^{\prime \prime \prime \prime}-2 A w^{\prime \prime}+B^{2} w=|w|^{q-2} w \quad \text { on } \mathbb{R}
$$

has at least a solution $w \in H^{2}(\mathbb{R})$. More precisely, $w$ achieves

$$
I(A, B):=\inf _{\substack{w \in H^{2}(\mathbb{R}) \\ w \neq 0}} \frac{\int\left(\left|w_{s s}\right|^{2}+2 A\left|w_{s}\right|^{2}+B^{2}|w|^{2}\right) d s}{\left(\int|w|^{q} d s\right)^{2 / q}} .
$$

(ii) If in addition $A \geq B$ then $w$ is the unique (up to translations, inversion $s \mapsto-s$ and change of sign) nontrivial solution to 2.4) in $H^{2}(\mathbb{R})$. Moreover, $w$ can be taken to be even, positive and strictly decreasing on $\mathbb{R}_{+}$. 
Proof. Existence follows by nowadays standard variational arguments, by showing that every minimizing sequence is relatively compact in $H^{2}(\mathbb{R})$ up to translations in $\mathbb{R}$ (use for instance the techniques in [11]).

Next, we assume that $A \geq B$. We first notice that $w$ has at least one critical point, since it is smooth and vanishes at infinity. The next claim is the main step in our argument:

$$
\text { if } w^{\prime}\left(s_{0}\right)=0 \text {, then } w \text { is even with respect to } s_{0} \text {. }
$$

To prove the claim, we first notice that we can assume that $s_{0}=0$. We put

$$
\eta(s):=w(s)-w(-s)
$$

We also define a measurable function $a(s)$ on $\mathbb{R}_{+}$as follows. In points $s$ where $\eta(s) \neq 0$ we set

$$
a(s):=\frac{|w(s)|^{q-2} w(s)-|w(-s)|^{q-2} w(-s)}{w(s)-w(-s)}
$$

and we put $a(s) \equiv 0$ on $\left\{\eta^{-1}(0)\right\}$. Notice that

$$
0 \leq a(s) \leq(q-1) \max \left\{|w(s)|^{q-2},|w(-s)|^{q-2}\right\} \quad \text { for any } s \in \mathbb{R}
$$

by the mean value theorem, so that in particular $a \in L^{\infty}\left(\mathbb{R}_{+}\right)$. Moreover, $\eta \in H^{2}(\mathbb{R})$ solves (2.1), with $a$ defined as above. Thus $\eta \equiv 0$ on $(0, \infty)$ by Lemma 2.1, that is, $w(s)=w(-s)$ for any $s>0$. Claim (2.6) is completely proved.

Proof of the Theorem concluded. Since any solution $w$ to (2.4) is smooth and vanishes at infinity, we can assume that $w$ attains its positive maximum at 0 . Moreover, 0 is the unique critical point of $w$. Indeed, assume that $w^{\prime}\left(s_{0}\right)=0$. Then both $s \mapsto w(s)$ and $s \mapsto w\left(s_{0}+s\right)$ are even by (2.6). Thus, for any $s \in \mathbb{R}$ we have that

$$
w\left(s+2 s_{0}\right)=w\left(s_{0}+\left(s_{0}+s\right)\right)=w\left(s_{0}-\left(s_{0}+s\right)\right)=w(-s)=w(s) .
$$

Hence $s_{0}=0$ since no function in $H^{2}(\mathbb{R})$ can be periodic. In particular, $w$ is strictly positive on $\mathbb{R}$ and decreasing on $\mathbb{R}_{+}$. Uniqueness then follows by the Amick and Toland theorem in [1. The proof is complete.

Remark 2.3 If $A, B$ satisfy

$$
\frac{A}{B}=\frac{q^{2}+4}{4 q}>1
$$

then the unique solution to (2.4) is given by

$$
w(s)=C(\cosh \nu s)^{-\frac{4}{q-2}},
$$

where

$$
\nu^{2}=\frac{(q-2)^{2}}{2\left(q^{2}+4\right)} A, \quad C^{q-2}=\frac{2 q(q+2)(3 q-2)}{\left(q^{2}+4\right)^{2}} A^{2} .
$$


Remark 2.4 By the results in [1], problem [2.4) has at most one homoclinic and positive solution for any $A, B>0$. However, if $A<B$ we do not know if there might exists nodal solutions $w \in H^{2}(\mathbb{R})$. Actually quite wild phenomena could appear. Consider the equation

$$
w^{\prime \prime \prime \prime}-2 A w^{\prime \prime}+B^{2} w=|w|^{2} \quad \text { on } \mathbb{R} .
$$

It is not difficult to show that (2.7) has a ground state solution $w \in H^{2}(\mathbb{R})$ for any $A, B>0$. Moreover, if $A \geq B$ then $w$ is positive, and hence it solves (2.4) for $q=3$. It suffices to introduce the root $c_{ \pm}>0$ to equation $c^{2}-2 A c+B^{2}=0$ as in the proof of Lemma 2.1 and to notice that $\psi:=-w^{\prime \prime}+c_{+} w$ solves $-\psi^{\prime \prime}+c_{-} \psi=|w|^{2} \geq 0$. Hence $-w^{\prime \prime}+c_{+} w \geq 0$, that implies $w \geq 0$.

On the other hand, it has been proved in [6] that a plethora (countably infinite set) of signchanging homoclinic solutions exists for $A / B<1$ close enough to 1 .

Remark 2.5 The differential equation in (2.4) is conservative, with Hamiltonian

$$
H\left(w, w^{\prime}, w^{\prime \prime}, w^{\prime \prime \prime}\right)=-w^{\prime \prime \prime} w^{\prime}+\frac{1}{2}\left|w^{\prime \prime}\right|^{2}+A\left|w^{\prime}\right|^{2}-\frac{1}{2} B^{2}|w|^{2}+\frac{1}{q}|w|^{q} .
$$

Therefore, any homoclinic solution $w$ to 2.4 satisfies

$$
-w^{\prime \prime \prime} w^{\prime}+\frac{1}{2}\left|w^{\prime \prime}\right|^{2}+A\left|w^{\prime}\right|^{2}-\frac{1}{2} B^{2}|w|^{2}+\frac{1}{q}|w|^{q} \equiv 0 \quad \text { on } \mathbb{R} .
$$

Using (2.8) one infers the following a-priori bound on homoclinic solutions to (2.4):

$$
\|w\|_{\infty}^{q-2} \leq \frac{q}{2} B^{2}
$$

\section{Radially symmetric solutions}

In this section we prove Theorems 1.1 and 1.2. The main tool is the Emden-Fowler transform, which has already been used in [4].

Given a radially symmetric function $u$ we define the Emden Fowler transform of $u$ by $w=T u$, where

$$
u(x)=|x|^{-\frac{N-4}{2}} w(-\log |x|) .
$$

It turns out that $T$ maps radially symmetric functions $u \in D^{2,2}\left(\mathbb{R}^{N}\right)$ into functions $w \in$ $H^{2}(\mathbb{R})$, and moreover

$$
\begin{gathered}
\int_{\mathbb{R}^{N}}|x|^{-\beta}|u|^{q} d x=\omega_{N} \int|w|^{q} d s \\
\int_{\mathbb{R}^{N}}|\Delta u|^{2} d x=\omega_{N} \int\left(\left|w_{s s}\right|^{2}+2\left(\gamma_{N}+2\right)\left|w_{s}\right|^{2}+\gamma_{N}{ }^{2}|w|^{2}\right) d s
\end{gathered}
$$

where $\omega_{N}=\left|\mathbb{S}^{N-1}\right|$ and $\gamma_{N}=N(N-4) / 4$, as in (1.3).

Proof of Theorem 1.1. From (3.1) and (3.2) it immediately follows that $u$ achieves $S_{q}^{\mathrm{rad}}(\lambda)$ if and only if $w=T u$ attains the infimum $I\left(\gamma_{N}+2, \gamma_{N}^{2}-\lambda\right)$, see (2.5). The conclusion follows from $(i)$ of Theorem 2.2 . 
Proof of Theorem 1.2, A direct computation shows that $u \in \mathcal{D}^{2,2}\left(\mathbb{R}^{N}\right)$ is a radial solution to (1.1) if and only if $T u=w \in H^{2}(\mathbb{R})$ is a solution to

$$
w^{\prime \prime \prime \prime}-2\left(\gamma_{N}+2\right) w^{\prime \prime}+\left(\gamma_{N}^{2}-\lambda\right) w=|w|^{q-2} w \quad \text { on } \mathbb{R} .
$$

Since $\lambda \geq-(N-2)^{2}$, then

$$
\left(\gamma_{N}+2\right)^{2}-\left(\gamma_{N}^{2}-\lambda\right)=4\left(\gamma_{N}+1\right)+\lambda=(N-2)^{2}+\lambda \geq 0
$$

The conclusion readily follows from the second part of Theorem 2.2 .

Remark 3.1 Using Remark [2.3, one can explicitly compute the radially symmetric solution to (1.1) when

$$
\lambda=\lambda(q):=\gamma_{N}^{2}-\left(\frac{4 q}{q^{2}+4}\right)^{2}\left(\gamma_{N}^{2}+2\right)^{2} .
$$

Notice that $\lambda(q)>-(N-2)^{2}$ for any $q \in\left(2,2^{* *}\right]$ and that $\lambda\left(2^{* *}\right)=0$. If $\lambda=\lambda(q)$ then the unique radially symmetric solution to (1.1) is given by

$$
u(x)=\widetilde{C}|x|^{\frac{4-N}{2}+\frac{4 \nu}{q-2}}\left(1+|x|^{2 \nu}\right)^{-\frac{4}{q-2}}
$$

for a computable constant $\widetilde{C}$ depending on $N, q$, where

$$
\nu=\frac{(q-2)^{2}}{2\left(q^{2}+4\right)}\left(\gamma_{N}^{2}+2\right) .
$$

The preliminary results in Section 2 can be applied to a larger class of fourth order equations in $\mathbb{R}^{N}$. Let us consider for instance a problem taken from [4].

Let $N \geq 2$ be any integer, and let $\alpha$ be a given exponent, such that

$$
\alpha>4-N, \quad \alpha \neq N
$$

By the results in [3], 4], it turns out that for any $q \in[2, \infty)$ the infimum

$$
S_{q, \alpha}^{\mathrm{rad}}:=\inf _{\substack{u \in \mathcal{N}_{\mathrm{rad}}^{2}\left(\mathbb{R}^{N} ; \alpha\right) \\ u \neq 0}} \frac{\int_{\mathbb{R}^{n}}|x|^{\alpha}|\Delta u|^{2} d x}{\left(\int_{\mathbb{R}^{n}}|x|^{-\beta_{\alpha}}|u|^{q} d x\right)^{2 / q}}
$$

is positive. Here

$$
\beta_{\alpha}:=N-q \frac{N-4+\alpha}{2}
$$

and $\mathcal{N}_{\text {rad }}^{2}\left(\mathbb{R}^{N} ; \alpha\right)$ is the completion of the space of radially symmetric functions in $C_{c}^{\infty}\left(\mathbb{R}^{N}\right)$ with respect to the Hilbertian norm

$$
\|u\|^{2}=\int_{\mathbb{R}^{N}}|x|^{\alpha}|\Delta u|^{2} d x .
$$


More precisely, for $q=2$ the radial Rellich constant is given by

$$
S_{2, \alpha}^{\mathrm{rad}}=\gamma_{N, \alpha}^{2}, \quad \text { where } \gamma_{N, \alpha}=\frac{(N-4+\alpha)(N-\alpha)}{4},
$$

and it is not achieved. In Theorem 2.8 of [4] it is proved that, for any $q>2$, the infimum $S_{2, \alpha}^{\text {rad }}$ is attained by some $u \in \mathcal{N}_{\text {rad }}^{2}\left(\mathbb{R}^{N} ; \alpha\right)$. Then, up to a Lagrange multiplier, $u$ solves

$$
\Delta\left(|x|^{\alpha} \Delta u\right)=|x|^{-\beta_{\alpha}}|u|^{q-2} u \quad \text { on } \mathbb{R}^{N} .
$$

We point out the following positivity and uniqueness result.

Theorem 3.2 Let $\alpha>4-N, \alpha \neq N$. Then problem (3.3) has a unique nontrivial solution $u \in \mathcal{N}_{\text {rad }}^{2}\left(\mathbb{R}^{N} ; \alpha\right)$. Moreover, $u>0$ on $\mathbb{R}^{N}$.

Proof. Existence follows from Theorem 2.8 in [4]. Following [3], we introduce the value

$$
\bar{\gamma}_{N, \alpha}=\left(\frac{N-2}{2}\right)^{2}+\left(\frac{\alpha-2}{2}\right)^{2}
$$

By direct computations (see also [3], 44), we have that $u \in \mathcal{N}_{\text {rad }}^{2}\left(\mathbb{R}^{N} ; \alpha\right)$ solves (3.3) if and only if $w \in H^{2}(\mathbb{R})$ solves

$$
w^{\prime \prime \prime \prime}-2 \bar{\gamma}_{N, \alpha} w^{\prime \prime}+\gamma_{N, \alpha}^{2} w=|w|^{q-2} w \quad \text { on } \mathbb{R} .
$$

Here we have denoted by $w=T_{\alpha} u$ the weighted Emden-Fowler transform of $u$ :

$$
u(x)=|x|^{\frac{4-N-\alpha}{2}} w(-\log |x|) .
$$

Since $\bar{\gamma}_{N, \alpha} \geq \gamma_{N, \alpha}$, then positivity and uniqueness follows by Theorem 2.2

\section{On the infimum $S_{q}(\lambda)$}

The following $\varepsilon$-compactness result is a basic tool in the proof of Theorem 1.3 ,

Proposition 4.1 Let $u_{n} \in \mathcal{D}^{2,2}\left(\mathbb{R}^{N}\right)$ such that $u_{n} \rightarrow 0$ in $\mathcal{D}^{2,2}\left(\mathbb{R}^{N}\right)$ and

$$
\begin{gathered}
\Delta^{2} u_{n}-\lambda|x|^{-4} u_{n}=|x|^{-\beta}\left|u_{n}\right|^{q-2} u_{n}+f_{n} \\
\int_{B_{R}}|x|^{-\beta}\left|u_{n}\right|^{q} d x \leq \varepsilon_{0} \quad \text { for some } \varepsilon_{0}, R>0,
\end{gathered}
$$

where $f_{n} \rightarrow 0$ in the dual space of $\mathcal{D}^{2,2}\left(\mathbb{R}^{N}\right)$. If $\varepsilon_{0}<S_{q}(\lambda)^{\frac{q}{q-2}}$, then

$$
|x|^{-\beta}\left|u_{n}\right|^{q} \rightarrow 0 \quad \text { in } L_{\mathrm{loc}}^{1}\left(B_{R}\right) .
$$


Proof. Fix any $R^{\prime} \in(0, R)$ and choose a cut off function $\phi \in C_{c}^{\infty}\left(B_{R}\right)$ such that $\phi \equiv 1$ in $B_{R^{\prime}}$. Since $\nabla \phi$ has compact support in $\mathbb{R}^{N} \backslash\{0\}$, then

$$
\int_{\mathbb{R}^{N}} \Delta u_{n} \Delta\left(\phi^{2} u_{n}\right) d x=\int_{\mathbb{R}^{N}}\left|\Delta\left(\phi u_{n}\right)\right|^{2} d x+o(1)
$$

by Rellich Theorem. Applying (4.1), Hölder inequality and (4.2) we obtain

$$
\begin{aligned}
\int_{\mathbb{R}^{N}}\left[\left|\Delta\left(\phi u_{n}\right)\right|^{2}-\lambda|x|^{-4}\left|\phi u_{n}\right|^{2}\right] d x & =\int_{\mathbb{R}^{N}}|x|^{-\beta}\left|u_{n}\right|^{q-2}\left|\phi u_{n}\right|^{2} d x \\
& \leq \varepsilon_{0}^{\frac{q-2}{q}}\left(\int_{\mathbb{R}^{N}}|x|^{-\beta}\left|\phi u_{n}\right|^{q} d x\right)^{\frac{2}{q}} .
\end{aligned}
$$

We estimate from below the left hand side of the above inequality by

$$
\int_{\mathbb{R}^{N}}\left[\left|\Delta\left(\phi u_{n}\right)\right|^{2}-\lambda|x|^{-4}\left|\phi u_{n}\right|^{2}\right] d x \geq S_{q}(\lambda)\left(\int_{\mathbb{R}^{N}}|x|^{-\beta}\left|\phi u_{n}\right|^{q} d x\right)^{2 / q}
$$

Thus

$$
S_{q}(\lambda)\left(\int_{\mathbb{R}^{N}}|x|^{-\beta}\left|\phi u_{n}\right|^{q} d x\right)^{2 / q} \leq \varepsilon_{0}^{\frac{q-2}{q}}\left(\int_{\mathbb{R}^{N}}|x|^{-\beta}\left|\phi u_{n}\right|^{q} d x\right)^{2 / q}
$$

Since $\varepsilon_{0}^{\frac{q-2}{q}}<S_{q}(\lambda)$ and $\phi \equiv 1$ on $B_{R^{\prime}}$, the conclusion follows by the arbitrariness of $R^{\prime} \in$ $(0, R)$.

\subsection{Proof of Theorem 1.3 in case $q<2^{* *}$}

Using Ekeland's variational principle we can fix a minimizing sequence for $S_{q}(\lambda)$ such that

$$
\begin{gathered}
\int_{\mathbb{R}^{N}}\left|\Delta u_{n}\right|^{2}-\lambda \int_{\mathbb{R}^{N}}|x|^{-4}\left|u_{n}\right|^{2}=\int_{\mathbb{R}^{N}}|x|^{-\beta}\left|u_{n}\right|^{q} d x+o(1) \\
=S_{q}(\lambda)^{\frac{q}{q-2}}+o(1) \\
\Delta^{2} u_{n}-\lambda|x|^{-4} u_{n}=|x|^{-\beta}\left|u_{n}\right|^{q-2} u_{n}+f_{n}
\end{gathered}
$$

where $f_{n} \rightarrow 0$ in the dual of $\mathcal{D}^{2,2}\left(\mathbb{R}^{N}\right)$. Up to a rescaling, we can also assume that

$$
\int_{B_{2}}|x|^{-\beta}\left|u_{n}\right|^{q} d x=\frac{1}{2} S_{q}(\lambda)^{\frac{q}{q-2}}
$$

Finally, since $\lambda<\gamma_{N}^{2}$, then $u_{n}$ is a bounded sequence in $\mathcal{D}^{2,2}\left(\mathbb{R}^{N}\right)$ by Rellich inequality. Therefore we can assume that there exists $u \in \mathcal{D}^{2,2}\left(\mathbb{R}^{N}\right)$ such that $u_{n} \rightarrow u$ weakly in $\mathcal{D}^{2,2}\left(\mathbb{R}^{N}\right)$. By standard arguments, to conclude it suffices to show that $u \neq 0$. We argue by contradiction. Suppose $u_{n} \rightarrow 0$. We can use Proposition (4.1) to get

$$
o(1)=\int_{B_{1}}|x|^{-\beta}\left|u_{n}\right|^{q} d x=\int_{B_{2}}|x|^{-\beta}\left|u_{n}\right|^{q} d x-\int_{1<|x|<2}|x|^{-\beta}\left|u_{n}\right|^{q} d x .
$$


Thus from (4.5) we infer

$$
\int_{1<|x|<2}|x|^{-\beta}\left|u_{n}\right|^{q} d x=\frac{1}{2} S_{q}(\lambda)^{\frac{q}{q-2}}+o(1)
$$

which readily leads to a contradiction by Rellich theorem, as $q \in\left(2,2^{* *}\right)$. Proposition $(i)$ in Theorem 1.3 is completely proved.

\subsection{Proof of Theorem 1.3 in the limiting case $q=2^{* *}$}

We start by pointing out a sufficient condition for existence.

Lemma 4.2 If $S_{2^{* *}}(\lambda)<S^{* *}$ then $S_{2^{* *}}$ is achieved.

Proof. As in the proof of part (i) we select a minimizing sequence for $S_{2^{* *}}(\lambda)$ satisfying

$$
\begin{gathered}
\int_{\mathbb{R}^{N}}\left|\Delta u_{n}\right|^{2} d x-\lambda \int_{\mathbb{R}^{N}}|x|^{-4}\left|u_{n}\right|^{2} d x=\int_{\mathbb{R}^{N}}\left|u_{n}\right|^{2^{* *}} d x+o(1) \\
=S_{2^{* *}}(\lambda)^{N / 4}+o(1) \\
\Delta^{2} u_{n}-\lambda|x|^{-4} u_{n}=\left|u_{n}\right|^{2^{* *}-2} u_{n}+f_{n} \\
\int_{B_{2}}\left|u_{n}\right|^{2^{* *}} d x=\frac{1}{2} S_{2^{* *}}(\lambda)^{N / 4},
\end{gathered}
$$

where $f_{n} \rightarrow 0$ in the dual of $\mathcal{D}^{2,2}\left(\mathbb{R}^{N}\right)$. In addition, we can assume that $u_{n}$ converges weakly in $\mathcal{D}^{2,2}\left(\mathbb{R}^{N}\right)$. Again, we only have to exclude that the weak limit vanishes. By contradiction, assume that $u_{n} \rightarrow 0$ in $\mathcal{D}^{2,2}\left(\mathbb{R}^{N}\right)$. Arguing as in the proof of part $(i)$ we can conclude that (4.6) holds with $q=2^{* *}$, that is,

$$
\int_{1<|x|<2}\left|u_{n}\right|^{2 *} d x=\frac{1}{2} S_{2^{* *}}(\lambda)^{N / 4}+o(1) .
$$

Now we choose $\phi$ to be a cut off function in $C_{c}^{\infty}\left(\mathbb{R}^{N} \backslash\{0\}\right)$ such that $\phi \equiv 1$ in $B_{2} \backslash B_{1}$. We use $\phi^{2} u_{n}$ as a test function in (4.7). Rellich theorem and Hölder inequality plainly lead to

$$
\int_{\mathbb{R}^{N}}\left[\left|\Delta\left(\phi u_{n}\right)\right|^{2}-\lambda|x|^{-4}\left|\phi u_{n}\right|^{2}\right] d x \leq S_{2^{* *}}(\lambda)\left(\int_{\mathbb{R}^{N}}\left|\phi u_{n}\right|^{2^{* *}} d x\right)^{\frac{N-4}{N}}+o(1)
$$

(argue as in the proof of part $(i)$ ). Since $\phi$ has compact support in $\mathbb{R}^{N} \backslash\{0\}$, then Rellich theorem again and the Sobolev inequality give

$$
\int_{\mathbb{R}^{N}}\left[\left|\Delta\left(\phi u_{n}\right)\right|^{2}-\lambda|x|^{-4}\left|\phi u_{n}\right|^{2}\right]=\int_{\mathbb{R}^{N}}\left|\Delta\left(\phi u_{n}\right)\right|^{2}+o(1) \geq S^{* *}\left(\int_{\mathbb{R}^{N}}\left|\phi u_{n}\right|^{2^{* *}}\right)^{2 / 2^{* *}} .
$$

In conclusion, we have proved that

$$
S^{* *}\left(\int_{\mathbb{R}^{N}}\left|\phi u_{n}\right|^{2^{* *}} d x\right)^{2 / 2^{* *}} \leq S_{2^{* *}}(\lambda)\left(\int_{\mathbb{R}^{N}} \mathbb{R}^{N}\left|\phi u_{n}\right|^{2^{* *}} d x\right)^{2 / 2^{* *}},
$$

which implies $\int_{\mathbb{R}^{N}}\left|\phi u_{n}\right|^{2^{* *}}=o(1)$. Hence $\int_{B_{2} \backslash B_{1}}\left|u_{n}\right|^{2^{* *}} d x=o(1)$, since $\phi \equiv 1$ in $B_{2} \backslash B_{1}$ This is a contradiction to (4.8). Thus $u \neq 0$ and achieves $S_{2 * *}(\lambda)$. 
Proof of Theorem 1.3- (ii). We start by recalling that the Sobolev constant $S^{* *}$ is achieved in $\mathcal{D}^{2,2}\left(\mathbb{R}^{N}\right)$, see for instance [14]. Next, let $u$ be any fixed function in $C_{c}^{\infty}\left(\mathbb{R}^{N} \backslash\{0\}\right)$, and put $v_{y}(x)=u(x+y)$. Since

$$
\begin{aligned}
S_{2^{* *}}(\lambda) & \leq \lim _{|y| \rightarrow \infty} \frac{\int\left[\left|\Delta v_{y}\right|^{2}-\lambda|x|^{-4}\left|v_{y}\right|^{2}\right]}{\left(\int_{\mathbb{R}^{N}}\left|v_{y}\right|^{2^{* *}}\right)^{2 / 2^{* *}}}=\lim _{|y| \rightarrow \infty} \frac{\int\left[|\Delta u|^{2}-\lambda|x-y|^{-4}|u|^{2}\right]}{\left(\int_{\mathbb{R}^{N}}|u|^{2 *}\right)^{2 / 2^{* *}}} \\
& =\frac{\int|\Delta u|^{2}}{\left(\int_{\mathbb{R}^{N}}|u|^{2^{* *}}\right)^{2 / 2^{* *}}},
\end{aligned}
$$

then $S_{2^{* *}}(\lambda) \leq S^{* *}$ for every $\lambda \in \mathbb{R}$. Now assume $\lambda<0$. Then $S_{2^{* *}}(\lambda) \geq S^{* *}$, that is, $S_{2^{* *}}(\lambda)=S^{* *}$. Therefore $S_{2^{* *}}(\lambda)$ can not be achieved since $S^{* *}$ is achieved.

Now assume $0<\lambda<\gamma_{N}^{2}$ and let $U$ be an extremal for $S^{* *}$. Then

$$
S_{2^{* *}}(\lambda) \leq \frac{\int_{\mathbb{R}^{N}}\left[|\Delta U|^{2}-\lambda|x|^{-4}|U|^{2}\right]}{\left(\int_{\mathbb{R}^{N}}|U|^{2 *}\right)^{2 / 2^{* *}}}<\frac{\int_{\mathbb{R}^{N}}|\Delta U|^{2}}{\left(\int_{\mathbb{R}^{N}}|U|^{2 *}\right)^{2 / 2^{* *}}}=S^{* *} .
$$

and hence $S_{2^{* *}}(\lambda)$ is achieved by Lemma 4.2,

\section{$5 \quad$ Positivity, symmetry and breaking symmetry}

In this section we study the symmetry and breaking symmetry of $S_{q}(\lambda)$ depending on the parameter $\lambda$. We identify functions $u$ that coincide up to a multiplicative constant and up to a transform of the kind (1.7).

We start by recalling that in case $\lambda=0$ and $q=2^{* *}$, the Sobolev constant $S^{* *}=S_{2}^{* *}(0)$ is achieved by the radially symmetric function

$$
U(x)=\left(1+|x|^{2}\right)^{\frac{4-N}{2}},
$$

see for instance [14]. Moreover, $u$ achieves $S^{* *}$ if and only if $u$ is the composition of $U$ with a translation in $\mathbb{R}^{N}$ (here we use the above identification). Notice that in particular $U$ is positive.

Since truncations $u \mapsto u^{ \pm}$are not allowed in dealing with fourth order differential operators, the positivity of extremals for $S_{q}(\lambda)$ does not follow by standard arguments.

In the first result we use rearrangement techniques and the uniqueness result in Theorem 1.2 to investigate the case $\lambda \geq 0$. 
Theorem 5.1 Assume $\lambda \neq 0$ or $q<2^{* *}$. If $\lambda \geq 0$ then $S_{q}(\lambda)$ is achieved by a unique function $u \in \mathcal{D}^{2,2}\left(\mathbb{R}^{N}\right)$. Moreover, $u$ is positive, radially symmetric about the origin and radially decreasing.

Proof. Let $u$ to be an extremal of $S_{q}(\lambda)$ and denote by $(-\Delta u)^{*}$ the Schwartz symmetrization of $-\Delta u$. We use Lemma A.1 in the appendix to introduce the function $v \in \mathcal{D}^{2,2}\left(\mathbb{R}^{N}\right)$ satisfying

$$
-\Delta v=(-\Delta u)^{*}
$$

In turns out that $u^{*} \leq v$ on $\mathbb{R}^{N}$, see for instance Remark II.13 in [11]. Clearly, if $u=u^{*}$ then we are done. Assume by contradiction that $u \neq u^{*}$. By the theory of symmetrisation (see Lieb and Loss [10], Theorem 3.4), we first obtain

$$
\int_{\mathbb{R}^{N}}|\Delta v|^{2} d x=\int_{\mathbb{R}^{N}}\left|(-\Delta u)^{*}\right|^{2} d x=\int_{\mathbb{R}^{N}}|\Delta u|^{2} d x .
$$

In addition, since we are assuming that $u^{*} \neq u$, then

$$
\int_{\mathbb{R}^{N}}|x|^{-4}|u|^{2} d x<\int_{\mathbb{R}^{N}}|x|^{-4}\left(|u|^{2}\right)^{*} d x=\int_{\mathbb{R}^{N}}|x|^{-4}\left|u^{*}\right|^{2} d x \leq \int_{\mathbb{R}^{N}}|x|^{-4}|v|^{2} d x .
$$

Thus we infer that

$$
\lambda \int_{\mathbb{R}^{N}}|x|^{-4}|u|^{q} d x \leq \lambda \int_{\mathbb{R}^{N}}|x|^{-4}|v|^{q} d x
$$

and that the strict inequality holds if $\lambda>0$. Similarly, we find

$$
\int_{\mathbb{R}^{N}}|x|^{-\beta}|u|^{q} d x \leq \int_{\mathbb{R}^{N}}|x|^{-\beta}|v|^{q} d x
$$

and the strict inequality holds if $\beta>0$, that is, if $q<2^{* *}$. In conclusion, since we are assuming that $\lambda$ and $\beta$ are not contemporarily zero, we have that

$$
S_{q}(\lambda) \leq \frac{\int_{\mathbb{R}^{N}}\left[|\Delta v|^{2}-\lambda|x|^{-4}|v|^{2}\right] d x}{\left(\int_{\mathbb{R}^{N}}|x|^{-\beta}|v|^{q} d x\right)^{2 / q}}<\frac{\int_{\mathbb{R}^{N}}\left[|\Delta u|^{2}-\lambda|x|^{-4}|u|^{2}\right] d x}{\left(\int_{\mathbb{R}^{N}}|x|^{-\beta}|u|^{q} d x\right)^{2 / q}}=S_{q}(\lambda),
$$

a contradiction. Therefore $u=u^{*}$, that is, $u$ is nonnegative and radially symmetric decreasing function.

Uniqueness follows immediately by using the Emden-Fowler transform as in Section 3 and Theorem 1.2 .

As soon as $\lambda \rightarrow-\infty$, a braking symmetry phenomenon appears. The next theorem applies in case $q<2^{* *}$, due to the nonexistence result pointed out in the critical case $q=2^{* *}, \lambda<0$. We quote [9], and [7] for remarkable breaking symmetry results for similar second-order equations. 
Theorem 5.2 If $\lambda<<0$ then $S_{q}(\lambda)<S_{q}^{\mathrm{rad}}(\lambda)$ and hence no extremal for $S_{q}(\lambda)$ is radially symmetric.

Proof. We already know that $S_{q}(\lambda) \leq S_{q}^{\mathrm{rad}}(\lambda)$. We will give an explicit condition on $\lambda$ to have $S_{q}(\lambda)<S_{q}^{\mathrm{rad}}(\lambda)$. Define

$$
n(u)=\int_{\mathbb{R}^{N}}\left[|\Delta u|^{2}-\lambda|x|^{-4}|u|^{2}\right] d x, \quad d(u)=\left(\int_{\mathbb{R}^{N}}|x|^{-\beta}|u|^{q} d x\right)^{2 / q}
$$

and $Q(u)=n(u) / d(u)$. Assume that $u$ is a radially symmetric minimizer of $Q$ on $\mathcal{D}^{2,2}\left(\mathbb{R}^{N}\right)$. Our goal is to show that $-\lambda$ can not be too large. By homogeneity we can assume that $d(u)=1$. Thus $Q^{\prime}(u) \cdot v=0$ and $Q^{\prime \prime}(u)[v, v] \geq 0$ for all $v \in \mathcal{D}^{2,2}\left(\mathbb{R}^{N}\right)$, that is, $n^{\prime}(u) \cdot v=$ $Q(u) d^{\prime}(u) \cdot v$ and

$$
n^{\prime \prime}(u)[v, v] \geq Q(u) d^{\prime \prime}(u)[v, v]
$$

for all $v \in \mathcal{D}^{2,2}\left(\mathbb{R}^{N}\right)$. We compute $n^{\prime \prime}(u)[v, v]=2 \int_{\mathbb{R}^{N}}\left(|\Delta v|^{2}-\lambda|x|^{-4}|v|^{2}\right)$ and

$$
d^{\prime \prime}(u)[v, v]=2(2-q)\left(\int_{\mathbb{R}^{N}}|x|^{-\beta}|u|^{q-2} u v\right)^{2}+2(q-1) \int_{\mathbb{R}^{N}}|x|^{-\beta}|u|^{q-2} v^{2} .
$$

Now we choose the test function $v$. Let $\varphi_{1} \in H^{1}\left(\mathbb{S}^{N-1}\right)$ be an eigenfunction of LaplaceBeltrami operator on $\mathbb{S}^{N-1}$ corresponding to the smallest positive eigenvalue. Thus

$$
-\Delta_{\sigma} \varphi_{1}=(N-1) \varphi_{1}, \frac{1}{\left|\mathbb{S}^{N-1}\right|} \int_{\mathbb{S}^{N-1}}\left|\varphi_{1}\right|^{2} d \sigma=1, \quad \int_{\mathbb{S}^{N-1}} \varphi_{1} d \sigma=0 .
$$

Define the test function $v$ as $v=u \varphi_{1}$. Thus

$$
d^{\prime \prime}(u)[v, v]=2(q-1) \int_{\mathbb{R}^{N}}|x|^{-\beta}|u|^{q}=2(q-1)
$$

and

$$
\begin{aligned}
\int_{\mathbb{R}^{N}}|\Delta v|^{2}= & \int_{\mathbb{R}^{N}}\left|\Delta\left(u \varphi_{1}\right)\right|^{2}=\left.\left.\int_{\mathbb{R}^{N}}|\Delta u-(N-1)| x\right|^{-2} u\right|^{2} \\
\leq & \int_{\mathbb{R}^{N}}\left[|\Delta u|^{2}+(N-1)^{2}|x|^{-4} u^{2}\right] \\
& \quad+2(N-1)\left(\int_{\mathbb{R}^{N}}|x|^{-4} u^{2}\right)^{1 / 2}\left(\int_{\mathbb{R}^{N}}|\Delta u|^{2}\right)^{1 / 2}
\end{aligned}
$$

by Hölder inequality. Therefore from (5.1) and from the definition of $Q(u)=n(u)$ we obtain

$$
\begin{aligned}
(q-2) \int_{\mathbb{R}^{N}}|\Delta u|^{2} \leq\left((N-1)^{2}+\lambda(q-2)\right) \int_{\mathbb{R}^{N}}|x|^{-4} u^{2} & \\
& +2(N-1)\left(\int_{\mathbb{R}^{N}}|x|^{-4} u^{2}\right)^{1 / 2}\left(\int_{\mathbb{R}^{N}}|\Delta u|^{2}\right)^{1 / 2} .
\end{aligned}
$$


In particular, the quantity

$$
X:=\left(\frac{\int_{\mathbb{R}^{N}}|\Delta u|^{2}}{\int_{\mathbb{R}^{N}}|x|^{-4} u^{2}}\right)^{1 / 2}
$$

satisfies the inequality

$$
(q-2) X^{2}-2(N-1) X-\left((N-1)^{2}+\lambda(q-2)\right) \leq 0,
$$

and hence it necessarily holds that

$$
\lambda \geq \min _{t \in \mathbb{R}}\left(t^{2}-\frac{2(N-1)}{q-2} t-\frac{(N-1)^{2}}{q-2}\right)=-\frac{(q-1)(N-1)^{2}}{(q-2)^{2}} .
$$

Thus, if

$$
\lambda<-\frac{(q-1)(N-1)^{2}}{(q-2)^{2}} .
$$

then no extremal for $S_{q}(\lambda)$ is radially symmetric and symmetry breaking occurs.

Remark 5.3 If $q$ is close enough to $2^{* *}$ then one can obtain a better estimate on the breaking symmetry parameter $\lambda$ by arguing as follows. Notice that $X>\gamma_{N}$ by the Rellich inequality (1.4). Thus, if $\gamma_{N} \geq \frac{N-1}{q-2}$ that is, if

$$
2+\frac{4(N-1)}{N(N-4)} \leq q \leq 2^{* *}
$$

then the radial solution $u$ does not achieve $S_{q}(\lambda)$ unless

$$
\lambda>\min _{t \geq \gamma_{N}}\left(t^{2}-\frac{2(N-1)}{q-2} t-\frac{(N-1)^{2}}{q-2}\right)=\gamma_{N}^{2}-\frac{N-1}{q-2}\left(N-1+2 \gamma_{N}\right) .
$$

Conversely, if

$$
\lambda \leq \gamma_{N}^{2}-\frac{N-1}{q-2}\left(N-1+2 \gamma_{N}\right)
$$

then breaking symmetry occurs.

\section{Appendix}

The following lemma has been used in the proof Theorem 5.1 (see also [11, Remark II.13).

Lemma A.1 Let $N \geq 5$ and $g \in L^{2}\left(\mathbb{R}^{N}\right)$. Then the equation

$$
-\Delta v=g \quad \text { on } \mathbb{R}^{N}
$$

has a unique solution $v \in \mathcal{D}^{2,2}\left(\mathbb{R}^{N}\right)$. 
Due to the unboundedness of the domain, Lemma A.1 does not follows by using a direct variational approach. Indeed, in general, the solution $v$ in Lemma A.1 does not belong to $\mathcal{D}^{1,2}\left(\mathbb{R}^{N}\right)$. For instance, for any $t \in[2,4)$ the smooth function

$$
v(x)=\left(1+|x|^{2}\right)^{\frac{t-N}{4}}
$$

satisfies $\Delta v \in L^{2}\left(\mathbb{R}^{N}\right)$ but $\int_{\mathbb{R}^{N}}|\nabla v|^{2} d x=\infty$.

Lemma A.1 can be proved by investigating the integrability properties of the Green's function at infinity. However, we will use here an alternative approach that allows us to underline the unexpected role of the Rellich inequality.

Actually, to better understand the phenomena involved we will consider a larger class of non-homogeneous linear problems on cones in $\mathbb{R}^{N}, N \geq 2$. We emphasize the fact that low dimensions are included. We first introduce some notation.

Let $\Sigma$ be a domain of class $C^{2}$ in the unit sphere $\mathbb{S}^{N-1}$. We introduce the cone

$$
\mathcal{C}_{\Sigma}:=\{r \sigma \mid r>0, \sigma \in \Sigma\}
$$

We denote by $\lambda_{\Sigma}$ the bottom of the Dirichlet spectrum of the Laplace-Beltrami operator on $\Sigma$. For instance, if $\Sigma=\mathbb{S}^{N-1}$ then $\mathcal{C}_{\mathbb{S}^{N-1}}=\mathbb{R}^{N} \backslash\{0\}$ and $\lambda_{\mathbb{S}^{N-1}}=0$. If $\Sigma$ is has compact closure in $\mathbb{S}^{N-1}$ then $\lambda_{\Sigma}>0$. Then the following Hardy inequality holds.

Lemma A.2 Let $\Sigma$ be a domain of class $C^{2}$ in the unit sphere $\mathbb{S}^{N-1}$, with $N \geq 2$. Then

$$
\int_{\mathcal{C}_{\Sigma}}|x|^{-2}|\nabla u|^{2} d x \geq\left[\left(\frac{N-4}{2}\right)^{2}+\lambda_{\Sigma}\right] \int_{\mathcal{C}_{\Sigma}}|x|^{-4}|u|^{2} d x
$$

for any $u \in C_{c}^{\infty}\left(\mathcal{C}_{\Sigma}\right)$.

Proof. Fix any $u \in C_{c}^{\infty}\left(\mathcal{C}_{\Sigma}\right)$. Use the divergence theorem and Proposition 1.1 in [8] to estimate

$$
\begin{aligned}
\int_{\mathcal{C}_{\Sigma}}|x|^{-2}|\nabla u|^{2} d x & =\int_{\mathcal{C}_{\Sigma}}\left|\nabla\left(|x|^{-1} u\right)\right|^{2} d x-(N-3) \int_{\mathcal{C}_{\Sigma}}|x|^{-4}|u|^{2} d x \\
& \geq\left[\left(\frac{N-2}{2}\right)^{2}+\lambda_{\Sigma}-(N-3)\right] \int_{\mathcal{C}_{\Sigma}}|x|^{-4}|u|^{2} d x \\
& =\left[\left(\frac{N-4}{2}\right)^{2}+\lambda_{\Sigma}\right] \int_{\mathcal{C}_{\Sigma}}|x|^{-4}|u|^{2} d x
\end{aligned}
$$

as desired. 
From now on we assume that

$$
\gamma_{N}+\lambda_{\Sigma}>0
$$

where $\gamma_{N}=N(N-4) / 4$, as before. Assumption (A.2) is satisfied if $\Sigma=\mathbb{S}^{N-1}$ and $N \geq 5$. In addition, the cases $N \geq 3$ and $\mathcal{C}_{\Sigma}=\mathbb{R}_{+}^{N}=$ homogeneous half-space are included, as the first Dirichlet eigenvalue of the Laplace-Beltrami operator on the half-sphere in $\mathbb{R}^{N}$ is $N-1$. In case $N=2$, assumption (A.2) is satisfied by any strictly convex cone.

If (A.2) holds, then in particular the Hardy constant in inequality (A.1) is positive, and we can define a Hilbert space $\mathcal{D}^{1,2}\left(\mathcal{C}_{\Sigma} ;|x|^{-2} d x\right)$ of maps $u$ vanishing on $\partial \mathcal{C}_{\Sigma}$ (if not empty), and such that

$$
\|u\|^{2}=\int_{\mathcal{C}_{\Sigma}}|x|^{-2}|\nabla u|^{2} d x<\infty .
$$

Then $\mathcal{D}^{1,2}\left(\mathcal{C}_{\Sigma} ;|x|^{-2} d x\right)$ is continuously embedded into $L^{2}\left(\mathcal{C}_{\Sigma} ;|x|^{-4} d x\right)$. Next we put

$$
\mathcal{N}^{2}\left(\mathcal{C}_{\Sigma}\right)=\left\{u \in \mathcal{D}^{1,2}\left(\mathcal{C}_{\Sigma} ;|x|^{-2} d x\right) \mid \Delta u \in L^{2}\left(\mathcal{C}_{\Sigma}\right)\right\}
$$

By the Rellich inequality on cones proved in [3] and using (A.2) we get that

$$
\int_{\mathcal{C}_{\Sigma}}|\Delta u|^{2} d x \geq\left(\gamma_{N}+\lambda_{\Sigma}\right)^{2} \int_{\mathcal{C}_{\Sigma}}|x|^{-4}|u|^{2} d x \quad \text { for any } u \in \mathcal{N}^{2}\left(\mathcal{C}_{\Sigma}\right)
$$

Thus $\mathcal{N}^{2}\left(\mathcal{C}_{\Sigma}\right)$ is a Hilbert space with norm

$$
\|u\|^{2}=\int_{\mathcal{C}_{\Sigma}}|\Delta u|^{2} d x
$$

and $\mathcal{N}^{2}\left(\mathcal{C}_{\Sigma}\right) \hookrightarrow L^{2}\left(\mathcal{C}_{\Sigma} ;|x|^{-4} d x\right)$.

A density argument can be used to show that $\mathcal{N}^{2}\left(\mathbb{R}^{N} \backslash\{0\}\right)=\mathcal{D}^{2,2}\left(\mathbb{R}^{N}\right)$ if $N \geq 5$. Thus the next proposition includes Lemma A.1 by taking $\Sigma=\mathbb{S}^{N-1}$.

Proposition A.3 Let $\Sigma$ be a domain of class $C^{2}$ in the unit sphere $\mathbb{S}^{N-1}$, and assume that $\gamma_{N}+\lambda_{\Sigma}>0$. Then for any $g \in L^{2}\left(\mathcal{C}_{\Sigma}\right)$ there exists a unique $v \in \mathcal{N}^{2}\left(\mathcal{C}_{\Sigma}\right)$ such that

$$
-\Delta v=g \quad \text { in } \mathcal{C}_{\Sigma}
$$

Moreover, $v$ satisfies the Navier boundary conditions

$$
v=\Delta v=0 \quad \text { on } \partial \mathcal{C}_{\Sigma}
$$

if $\Sigma$ is properly contained in $\mathbb{S}^{N-1}$

Proof. We only have to prove existence. Uniqueness easily follows. For any $R>1$ we put

$$
A_{R}^{\Sigma}=\left\{r \sigma \mid R^{-1}<r<R, \sigma \in \Sigma\right\} .
$$


Let $v_{R} \in H_{0}^{1}\left(A_{R}^{\Sigma}\right)$ be the unique solution to

$$
\begin{cases}-\Delta v_{R}=g & \text { in } A_{R}^{\Sigma} \\ v=0 & \text { on } \partial A_{R}^{\Sigma} .\end{cases}
$$

We denote by $v_{R} \in \mathcal{D}^{1,2}\left(\mathcal{C}_{\Sigma} ;|x|^{-4} d x\right)$ the null extension of $v_{R}$. Our aim is to use $|x|^{-2} v_{R}$ as test function in (A.3). Notice that

$$
\begin{aligned}
\int_{A_{R}^{\Sigma}}\left(-\Delta v_{R}\right)|x|^{-2} v_{R} & =\int_{A_{R}^{\Sigma}}|x|^{-2}\left|\nabla v_{R}\right|^{2} d x+\frac{1}{2} \int_{A_{R}^{\Sigma}} \nabla\left(|x|^{-2}\right) \cdot \nabla\left|v_{R}\right|^{2} d x \\
& =\int_{A_{R}^{\Sigma}}|x|^{-2}\left|\nabla v_{R}\right|^{2} d x+(N-4) \int_{A_{R}^{\Sigma}}|x|^{-4}\left|v_{R}\right|^{2} d x
\end{aligned}
$$

and therefore from (A.3) we get

$$
\begin{aligned}
\left(\int_{A_{R}^{\Sigma}}|g|^{2} d x\right)^{1 / 2}\left(\int_{A_{R}^{\Sigma}}|x|^{-4}\left|v_{R}\right|^{2} d x\right)^{1 / 2} & \\
& \geq \int_{A_{R}^{\Sigma}}|x|^{-2}\left|\nabla v_{R}\right|^{2} d x+(N-4) \int_{A_{R}^{\Sigma}}|x|^{-4}\left|v_{R}\right|^{2} d x \\
& \geq\left(\gamma_{N}+\lambda_{\Sigma}\right) \int_{A_{R}^{\Sigma}}|x|^{-4}\left|v_{R}\right|^{2} d x
\end{aligned}
$$

by the Cauchy-Schwarz inequality and by Lemma A.2. Since $\gamma_{N}+\lambda_{\Sigma}>0$ by assumption, we first infer that $v_{R}$ is bounded in $L^{2}\left(\mathcal{C}_{\Sigma} ;|x|^{-4} d x\right)$. Thus, using (A.4) again, we conclude that $v_{R}$ is uniformly bounded in $\mathcal{D}^{1,2}\left(\mathcal{C}_{\Sigma} ;|x|^{-2} d x\right)$. Therefore, up to a sequence $R \rightarrow \infty$, we can assume that $v_{R} \rightarrow v$ weakly in $\mathcal{D}^{1,2}\left(\mathcal{C}_{\Sigma} ;|x|^{-2} d x\right)$. It is easy to prove that $v \in$ $\mathcal{D}^{1,2}\left(\mathcal{C}_{\Sigma} ;|x|^{-2} d x\right) \hookrightarrow L^{2}\left(\mathcal{C}_{\Sigma} ;|x|^{-4} d x\right)$ solves $-\Delta v=g$ on $\mathcal{C}_{\Sigma}$. Thus in particular $\Delta v=-g \in$ $L^{2}\left(\mathcal{C}_{\Sigma}\right)$, that implies $v \in \mathcal{N}^{2}\left(\mathcal{C}_{\Sigma}\right)$. In case $\partial \mathcal{C}_{\Sigma}$ is not empty, then $v$ satisfies Navier boundary conditions by standard arguments.

Acknowledgements. This research was done when the first author was visiting the ICTP Mathematics Section in Trieste, Italy, and travel grant was sponsored by National Board of Higher Mathematics (NBHM), India. Warm hospitality of ICTP is gratefully acknowledged.

The second Author wishes to thank Prof. Fabio Zanolin for many helpful discussions about equation (2.4) and for having suggested the references [1, 6].

\section{References}

[1] Amick, C.J., Toland J.F., Global uniqueness of homoclinic orbits for a class of fourth order equations, ZAMP 43 (1992), 590-597.

[2] Caffarelli, L., Kohn, R., Nirenberg, L., First Order Interpolation Inequalities with Weight, Compositio Math. 53 (1984), 259-275. 
[3] Caldiroli, P., Musina, R., Rellich inequalities with weights. Calc. Var. PDE to appear (2011).

[4] Caldiroli, P., Musina, R., Caffarelli-Kohn-Nirenberg type inequalities for the weighted biharmonic operator in cones, Milan J. Math. 79 (2011), 657-687.

[5] Catrina, F., Wang, Z.-Q., On the Caffarelli-Kohn-Nirenberg inequalities: sharp constants, existence (and nonexistence), and symmetry of extremal functions, Comm. Pure Appl. Math. 54 (2001), 229258.

[6] Champneys, A.R., Toland, J.F., Bifurcation of a plethora of multi-modal homoclinic orbits for autonomous Hamiltonian systems, Nonlinearity 6 (1993), 665-721.

[7] Dolbeault, J., Esteban, M., Loss, M., Tarantello, G., On the symmetry of extremals for the CaffarelliKohn-Nirenberg inequalities, Adv. Nonlinear Stud. 9 (2009), 713-726.

[8] Fall, M.M., Musina, R., Hardy-Poincaré inequalities with boundary singularities, Proc. Roy. Soc. Edinburgh Sect. A, to appear.

[9] Felli, V., Schneider, M., Perturbation results of critical elliptic equations of Caffarelli-Kohn-Nirenberg type, J. Diff. Eq. 191 (2003), 121-142.

[10] Lieb, H.E., Loss, M., Analysis, Second Edition, Graduate Studies in Mathematics Vol 14, American Mathematical Society, 2001.

[11] Lions, P.L., The concentration-compactness principle in the calculus of variations. The locally compact case. II. Ann. Inst. H. Poincaré Anal. Non Linéaire 1 (1984), 223-283.

[12] Rellich, F., Halbbeschränkte Differentialoperatoren höherer Ordnung. In: J.C.H. Gerretsen, J. de Groot (Eds.): Proceedings of the International Congress of Mathematicians 1954, Volume III (pp. 243-250) Groningen: Noordhoff 1956.

[13] Rellich, F., Perturbation theory of eigenvalue problems, Gordon and Breach, New York, 1969.

[14] Swanson C.A., The best Sobolev constant, Appl. Anal. 47 (1992), 227-239.

[15] Terracini, S., On positive entire solutions to a class of equations with a singular coefficient and critical exponent, Adv. Diff. Eq. 2 (1996), 241-264. 